\title{
Hysteroscopic metroplasty of a T-shape uterus with intrauterine adhesions using blunt spreading dissection and ploughing techniques
}

\author{
Lingxiao Zou, Huan Huang, Aiqian Zhang, Arvind Burjoo, Fei Zeng, Dabao Xu \\ Department of Obstetrics and Gynecology, Third Xiangya Hospital of Central South University, Changsha 410013, China \\ Correspondence to: Fei Zeng, MD; Dabao Xu, MD. Department of Obstetrics and Gynecology, Third Xiangya Hospital of Central South University, \\ 138 Tongzipo Rd., Changsha 410013, China. Email: 444838636@qq.com; dabaoxu@yahoo.com.
}

Submitted Dec 30, 2019. Accepted for publication Jan 14, 2020.

doi: $10.21037 /$ atm.2020.01.133

View this article at: http://dx.doi.org/10.21037/atm.2020.01.133

\section{Clinical vignette}

A 30-year-old, gravida 3, para 0, abortus 2 woman suffered from amenorrhea after dilatation and curettage (D\&C) of a missed abortion at 2-month gestation. She had a mild cyclic lower abdominal pain before admission, and the estrogen/ progestogen challenge test was negative. The 3 -dimensional transvaginal ultrasound image (Figure 1) revealed a T-shaped uterine cavity with intrauterine adhesions (IUAs). The thickness of the left and right lateral wall was 22 and $23 \mathrm{~mm}$ respectively, and the fundal uterine wall thickness was $10 \mathrm{~mm}$. The T-shaped uterus with IUAs was diagnosed preoperatively.

\section{Surgical techniques}

\section{Preparation}

The patient was placed in the lithotomy position, and the disinfection and draping procedures were performed before intravenous general anesthesia administration.

\section{Exposition}

An anteverted uterus was confirmed by bimanual palpation. Exposure and disinfection of the cervix were performed. Normal saline was used as the distention medium with a distention pressure of $120 \mathrm{mmHg}$ and a flow rate of $350 \mathrm{~mL} / \mathrm{min}$.

\section{Operation}

Diagnostic and therapeutic hysteroscopy was performed using a 5.4-mm outer sheath hysteroscope with a 5-Fr working channel. The internal cervical os was completely occluded by adhesive tissue (Figure 2). With the magnifying effect of the hysteroscope, the layer between the anterior and posterior walls of the uterine cavity (Figure 3) could be seen clearly. Therefore, a 5-Fr double-action forceps was used to bluntly spread adhesions and find the original uterine cavity, known as the "blunt spreading dissection" technique (1). The key skill of this technique is opening the forceps in the direction toward the anterior and posterior walls of the uterus, or sometimes in the direction toward the left and right walls, to help separate adhesions.

The middle and lower uterine cavity was too narrow to enter due to the contraction of the adhesive tissue and the malformed bilateral walls. Therefore, a 5-Fr scissors was chosen to dissect the adhesions in the middle and lower uterine cavity, and plough the scars on the anterior and posterior walls, which is known as the "ploughing" technique (2). This technique facilitates the entry of the hysteroscope into the upper segment of the uterine cavity. A 5-Fr scissors was also used to restore the inverted triangle shape of the uterine cavity, in other words, the ipsilateral fallopian tubal ostium could be seen when the hysteroscope receded to the lower segment of the cavity. We continued to use the ploughing technique to correct the left and right uterine walls, and plough the fibrotic tissue on the anterior and posterior walls until the surface of the myometrial layer (Figure 4). A uterine-shaped intrauterine device (IUD) was inserted into the uterine cavity after the cavity had been corrected. It was necessary to perform hysteroscopy immediately to adjust the IUD position and ensure its 
Page 2 of 3

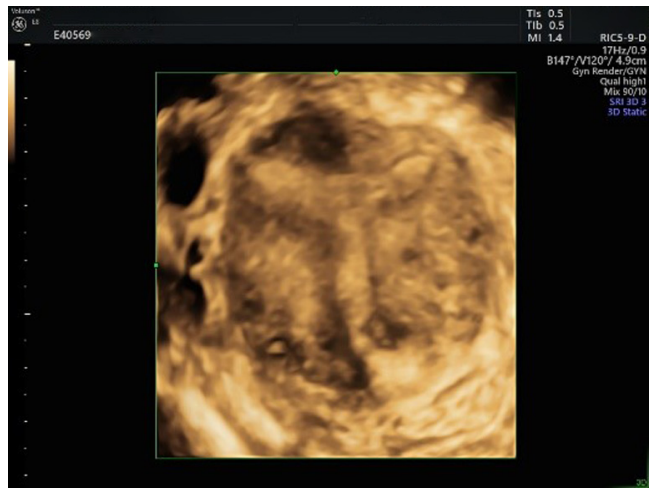

Figure 1 The three-dimensional transvaginal ultrasound image.

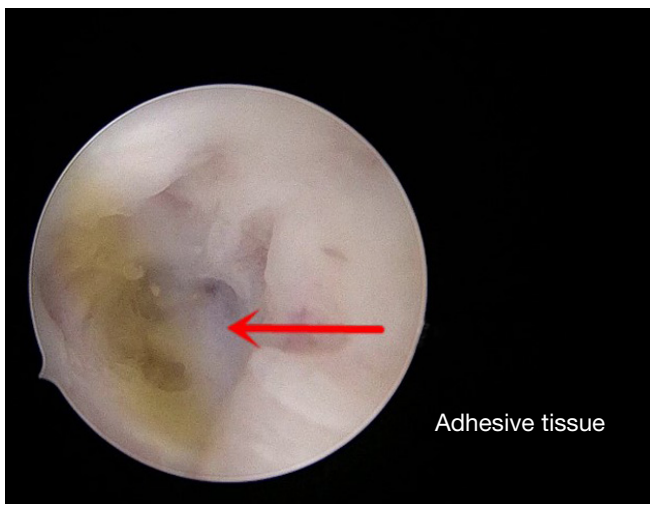

Figure 2 The internal cervical os was completely occluded by adhesive tissue.

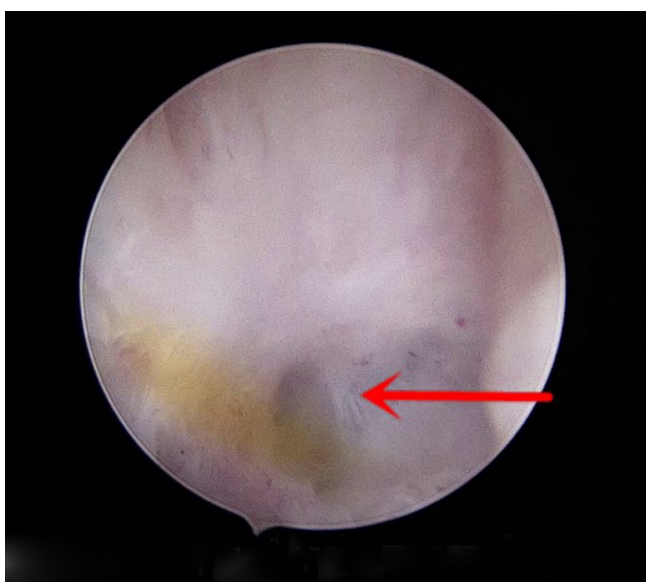

Figure 3 The layer between the anterior and posterior walls of the uterine cavity.

\section{Zou et al. Hysteroscopic metroplasty of a T-shape uterus with IUAs}

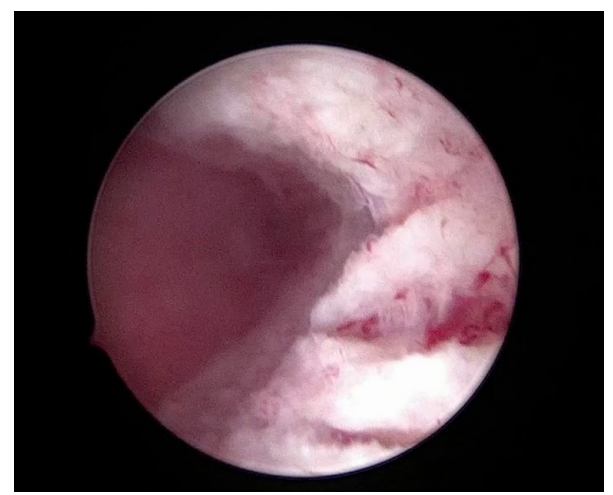

Figure 4 The wound after ploughing the fibrotic tissue on the posterior walls until the surface of the myometrial layer.

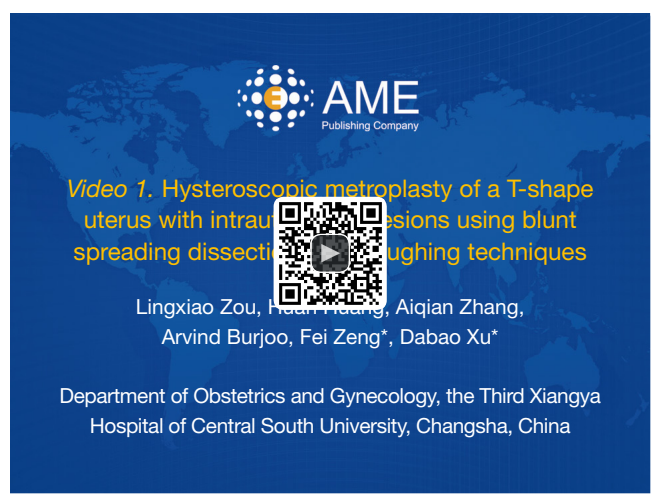

Figure 5 Hysteroscopic metroplasty of a T-shape uterus with intrauterine adhesions using blunt spreading dissection and ploughing techniques (3).

Available online: http://www.asvide.com/watch/33100

proper size. The procedure of hysteroscopic metroplasty is shown in the Figure 5.

\section{Completion}

At the end of the operation, $2 \mathrm{~mL}$ of the hyaluronic acid gel was injected into the uterine cavity, and a 12-Fr Foley catheter was also placed in it for 3 weeks. A following hysteroscopy was performed 1 month later.

\section{Comments}

\section{Clinical results}

The uterine cavity was corrected successfully, and there 
were no complications such as uterine perforation or fluid overload. Postoperative second-look hysteroscopy at 1 month revealed that there was no reformation of adhesions and an adequate uterine cavity was achieved.

\section{Advantages}

This was the first time we have used the ploughing technique to deal with the fibrotic tissue on the surface of the corrected T-shape uterine walls. It has proven to be beneficial in creating more space inside the uterine cavity and reducing the risk of the uterine cavity re-narrowing caused by contraction of fibrotic tissue postoperatively. Using the ploughing technique to divide the scars is also beneficial in creating a larger surface area inside the uterine cavity, thereafter, to provide more abundant blood supply for the growth of endometrium. When the internal cervical os is occluded, using the blunt spreading dissection technique helps find the correct layer of the uterine cavity while avoiding the creation of a false passage. Using the hysteroscope with a 5-Fr working channel for the diagnosis and treatment of hysteroscopic metroplasty under direct hysteroscopic vision can be performed in a onestep procedure without prior dilatation and hysteroscope changing, this is known as the "see and treat" technique. It lowers the risk of uterine perforation caused by blind dilatation. Moreover, the thermal and electrical injury to the endometrium was prevented owing to the use of cold forceps and scissors.

\section{Caveats}

By using a slim hysteroscope with 5-Fr forceps and scissors, blunt spreading dissection, and ploughing techniques, hysteroscopic metroplasty of a T-shape uterus with IUAs is a simple, effective, and safe performance. Preoperative 3-dimensional transvaginal ultrasound is helpful for the diagnosis of T-shaped uterus, and the assessment of the uterine cavity shape. Intraoperatively, retrieval and identification of the fallopian tubal ostia is essential because

Cite this article as: Zou L, Huang H, Zhang A, Burjoo A, Zeng F, Xu D. Hysteroscopic metroplasty of a T-shape uterus with intrauterine adhesions using blunt spreading dissection and ploughing techniques. Ann Transl Med 2020;8(4):60. doi: 10.21037/atm.2020.01.133 they are the anatomical markers during the hysteroscopic metroplasty.

\section{Acknowledgments}

Funding: This study was supported by the Hunan Science and Technology Department (grant no. 2018SK2102) and the Changsha Science and Technology Bureau (grant no. kq1901124).

\section{Footnote}

Conflicts of Interest: The authors have no conflicts of interest to declare.

Etbical Statement: The authors are accountable for all aspects of the work in ensuring that questions related to the accuracy or integrity of any part of the work are appropriately investigated and resolved. Approval was given to the study by The Institutional Review Board (IRB) of the Third Xiangya Hospital of Central South University. The procedure was performed in accordance with relevant guidelines and regulations. Informed consent was obtained after the procedure was fully explained to all participants and their legal guardians.

\section{References}

1. Huang H, Cheng C, Johnson G, et al. Hysteroscopic Intrauterine Adhesiolysis Using a Blunt Spreading Dissection Technique With Double-action Forceps. J Minim Invasive Gynecol 2018;25:583-4.

2. Zhang A, Jamail G, Xue M, et al. Hysteroscopic Intrauterine Adhesiolysis Using the "Ploughing" Technique With Cold Scissors. J Minim Invasive Gynecol 2015;22:934-5.

3. Zou L, Huang H, Zhang A, et al. Hysteroscopic metroplasty of a T-shape uterus with intrauterine adhesions using blunt spreading dissection and ploughing techniques. Asvide 2020;7:060. Available online: http:// www.asvide.com/watch/33100 